\section{PSICOLOGIA IBEROAMERICANA}

\section{Psicología lberoamericana}

ISSN: 1405-0943

psicología.iberoamericana@uia.mx

Universidad Iberoamericana, Ciudad de

México

México

Osés Bargas, Rosa María; Aguayo Chan, Jorge Carlos; Duarte Briceño, Efraín; Manuel Ortega, Jorge Isaac

Autorregulación en Estudiantes de Secundaria: Influencia de Sexo, Edad y Apoyo Familiar

Psicología Iberoamericana, vol. 19, núm. 2, julio-diciembre, 2011, pp. 38-46

Universidad Iberoamericana, Ciudad de México

Distrito Federal, México

Disponible en: http://www.redalyc.org/articulo.oa?id=133921440005

Cómo citar el artículo

- Número completo

- Más información del artículo

- Página de la revista en redalyc.org

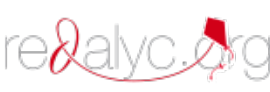

Sistema de Información Científica

Red de Revistas Científicas de América Latina, el Caribe, España y Portugal

Proyecto académico sin fines de lucro, desarrollado bajo la iniciativa de acceso abierto 


\title{
Autorregulación en Estudiantes de Secundaria: Influencia de Sexo, Edad y Apoyo Familiar
}

\author{
Self-regulation in Secondary Students: Gender, Age, \\ and Family Support Influence
}

\author{
Rosa María Osés Bargas \\ Jorge Carlos Aguayo Chan \\ Efraín Duarte Briceño \\ Jorge Isaac Manuel Ortega \\ Universidad Autónoma de Yucatán, México*
}

\section{RESUMEN}

Este trabajo evalúa la influencia de las variables de sexo, edad y apoyo familiar, tanto en la percepción que tienen los estudiantes del tiempo en que reciben apoyo en sus tareas escolares, como de los familiares que los ayudan en las mismas, en función de las estrategias autorregulatorias. Participaron 226 estudiantes del primer grado de una secundaria pública. Se usó una modificación del Cuestionario de Autorregulación de Flores Macías y Cerino Soberanis (2000), con cuatro componentes que miden: Estrategias de apoyo, Estrategias meta-cognitivas, Estrategias cognitivas y Motivación. Es una investigación aplicada, con un diseño Pretest-Postest de series de tiempo. Los resultados muestran que hubo un decremento en la motivación de las mujeres comparadas con los hombres. En las Estrategias de apoyo, los estudiantes de 12 años tuvieron ventaja sobre las demás edades, asimismo, hay una mejoría en las Estrategias cognitivas cuando el padre participa y cuando el apoyo son dos horas.

Descriptores: Autorregulación, estrategias cognitivas, motivación, apoyo familiar, secundaria.

\section{ABSTRACT}

This work evaluated the influences of the variables of gender, age, and time and family support student's perception with respect to the self-regulation strategies. 226 students from the first grade of a public secondary school participated. The Flores Macías and Cerino Soberanis (2000) Self-regulation Questionnaire modification was used to measure four components: Support strategies, Metacognitives strategies, Cognitives strategies and Motivation. This is an applied research with a pretest-postest design of times series. The outcomes showed that was a decrease in woman motivation compared with the man. In the support strategies, the 12 years old students had an advantage over the other ages, furthermore, there was an improvement in the cognitive strategies when the father participated, and when the time support were two hours.

Key words: Self-regulation, cognitive strategies, motivation, family support, high school 


\section{INTRODUCCIÓN}

La adolescencia es una etapa de desarrollo que ofrece a los jóvenes una gran cantidad de oportunidades para crecer, no sólo en el aspecto físico sino también en los aspectos cognoscitivos, sociales, de autonomía, autoestima e intimidad. Los cambios en la manera en cómo los jóvenes procesan la información varía mucho; sin embargo, los investigadores del procesamiento de información han identificado dos aspectos importantes en su cognición: cambios estructurales, que tienen que ver con el aumento de la capacidad de la memoria de trabajo y la memoria a largo plazo, y cambios funcionales, que se relacionan con el aprendizaje, el recuerdo y el razonamiento que mejoran en esta etapa. No obstante, estos progresos observados dependen de la motivación y la regulación emocional que experimenten estos jóvenes. Del mismo modo, sus logros escolares obedecen a las prácticas de crianza, el nivel socioeconómico, la calidad del ambiente familiar, el sexo, el origen étnico, la influencia de los pares, la calidad de la educación y la confianza en sí mismos (Papalia, Wendkos \& Duskin, 2010).

Las teorías que los padres tienen acerca de la crianza y el desarrollo infantil, reflejo de sus valores culturales y educativos, así como de los cambios vertiginosos de las tecnologías de la información que influyen en sus hijos, afectan las interacciones parentales que se presentan en el núcleo familiar, y los conflictos y las discusiones entre padres e hijos se agudizan más durante la adolescencia. Para poder entender estos cambios que se dan en esta etapa de la vida, se requiere analizar las condiciones sociales, culturales y económicas, variables contextuales que juegan un papel importante por su influencia sobre la familia y el individuo, principalmente en este mundo globalizado de tantos movimientos migratorios, sociales, demográficos, y con el uso de nuevas tecnologías (Oliva Delgado y Parra Jiménez, 2004). La familia juega un papel muy importante en el desarrollo de la personalidad de los jóvenes, así como en la socialización, porque cualquier estilo educativo puede potenciar o dificultar la individualización, la autonomía, la identidad, la confianza en sí mismo y el rendimiento académico de los adolescentes (González, 2006). Por lo tanto, el bajo rendimiento en todos los niveles escolares, y especialmente la falta de entrenamiento de habilidades de aprendizaje en los estudios de enseñanza primaria y secundaria, las diferencias estratégicas entre los estudiantes de éxito y de bajo rendimiento, o entre expertos y principiantes, han contribuido a un auge de estudios sobre el impacto de las estrategias de aprendizaje.

También hay que mencionar que muchas investigaciones reflejan que las relaciones familiares atraviesan por un periodo de desajuste y de mayor conflicto durante la adolescencia temprana, sobre todo en la comunicación entre padres e hijos, que suele deteriorarse en algún momento de la infancia y la adolescencia (Oliva Delgado y Parra Jiménez, 2004). El sexo parece influir sobre los patrones de comunicación familiar, ya que hay algunos estudios que demuestran que las jóvenes tienen una comunicación más frecuente con sus padres que los varones; sin embargo, parece ser que el sexo del progenitor es una variable importante para tener una comunicación más frecuente e íntima, siendo las madres las que mejor se comunican, tal vez por tener mayor disponibilidad de tiempo y porque se les percibe como más abiertas y comprensivas (Oliva Delgado y Parra Jiménez, 2004).

Otras investigaciones sobre el grado de involucramiento de los padres en las diferentes etapas del desarrollo de sus hijos señalan la importancia del papel de la familia en la crianza de los hijos y cómo las actitudes de los progenitores influyen en los distintos niveles de desarrollo. Por ejemplo, estudios realizados con padres latinos, en comparación con padres de otras culturas, demuestran que pasan más tiempo al cuidado de sus hijos, juegan más con ellos que las madres y muestran niveles similares de afecto y calidez que los padres de otras culturas (Parke, Dennis, Flyr, Morris, Leidy \& Schofield, 2005).

En un estudio realizado por Torres Velázquez y Rodríguez Soriano (2006) en México, acerca del rendimiento académico y el contexto familiar en estudiantes universitarios, se encontró una relación entre el apoyo que los estudiantes perciben de sus familias y su ejecución académica, además de que es un factor de suma importancia incluir a la familia para promover y elevar el rendimiento académico.

En un estudio sobre la visión de los padres y la visión de los hijos sobre las prácticas de crianza -realizado por Flores Galaz, Cortés Ayala y Góngora Coronado en primarias públicas del estado de Yucatán 
en 2008-, se encontró que las madres se perciben más afectuosas y apoyan más a sus hijos en las actividades escolares que los padres.

Otro estudio sobre el grado de participación de padres y madres de niños de primaria en las actividades educativas de sus hijos -realizado también en Yucatán, por Valdés Cuervo, Martín Pavón y Sánchez Escobedo en 2009-, mostró que la participación de los padres era baja o precaria, especialmente en el factor de comunicación y conocimiento de la escuela, mientras que las madres tuvieron un nivel de participación mayor en ambos factores, evidenciando que las encargadas de establecer el puente entre la escuela y la familia son las madres, ya que son quienes proporcionan los apoyos educativos a los hijos en casa.

Esto último concuerda conlos resultados de un estudio realizado en estudiantes de tercero de secundaria de una escuela pública de Mérida, sobre las estrategias de aprendizaje y calidad del desempeño académico, donde se encontró que, en cuanto a la percepción que tienen los estudiantes en relación con la ayuda recibida de sus padres en las tareas escolares, son las madres las que más apoyo les dan y quienes están más interesadas en que continúen sus estudios (Osés Bargas, Aguayo Chan, Duarte Briceño y Ortega, 2009).

Después de describir investigaciones que evidencian la importancia del involucramiento de la familia, vale revisar otros factores que favorecen un buen desempeño académico de los estudiantes en las aulas, por lo que es pertinente revisar la nueva concepción del aprendizaje; ésta concibe al estudiante como un ser activo que construye sus propios conocimientos, es decir, pone en contacto las habilidades del pensamiento con los datos informativos. En este sentido, y desde el punto de vista del aprendizaje y entrenamiento con medios naturales, es necesario estimular la autonomía y la capacidad de asumir de forma progresiva el control del aprendizaje (Beltrán Llera, 2004).

Las estrategias de aprendizaje son reglas o procedimientos que permiten tomar decisiones adecuadas en cualquier momento del proceso. Son las operaciones mentales que el estudiante lleva a cabo para facilitar y mejorar su tarea si selecciona, organiza y elabora los conocimientos. Implican un plan de acción, mientras que las técnicas son más mecánicas y rutinarias. Las estrategias tienen varios rasgos que las distinguen: su carácter intencional y propositivo, el de la conciencia, la selectividad y la autonomía. Como están directamente relacionadas con la calidad del aprendizaje del estudiante, permiten identificar y diagnosticar las causas del bajo o alto rendimiento escolar. Ofrecen un nuevo tipo de tecnología a la educación, además de que promueven un aprendizaje autónomo, independiente, de manera que las riendas y el control del aprendizaje vayan pasando del profesor a los estudiantes (Beltrán Llera, 2004).

Diversos autores han propuesto distintas taxonomías de estrategias de aprendizaje (Boekaerts, Pintrich \& Zeidner, 2000; Castañeda Figueiras y Ortega, 2004; Sternberg \& Baron, 1987), las cuales comparten algunos elementos y tienen una estructura semejante. En particular, en este trabajo se utilizará la de Beltrán Llera (2004), quien opina que las estrategias se pueden dividir según su naturaleza y función; así, fusionando los dos criterios, se pueden obtener cuatro grupos: apoyo, procesamiento, personalización y meta-cognitivas.

Las estrategias de apoyo están al servicio de la sensibilización del estudiante hacia las tareas de aprendizaje, la cual tiene entre sus ámbitos a la motivación, las actitudes y el afecto. Las de procesamiento, llamadas de adquisición, elaboración o construcción del conocimiento, son la repetición, selección, organización y elaboración. Las estrategias de personalización o cognitivas transforman la información, de manera que resulte en un conocimiento capaz de modificar la realidad, y están relacionadas con la creatividad, el pensamiento crítico y la capacidad de aplicar los conocimientos adquiridos en un contexto educativo (transfer). Las estrategias meta-cognitivas, planifican y supervisan la acción de las cognitivas, además de que ayudan al estudiante a conocer lo que sabe de la tarea, la naturaleza y el grado de dificultad de la misma; la forma de resolverla y el ambiente más favorable para enfrentarse a ella.

El enfoque cognitivo, en particular el de la corriente constructivista centrada en el estudiante, prioriza la participación del alumno como responsable de su propio aprendizaje, cuya meta es construir aprendizajes significativos, permanentes, aplicables y de fácil acceso, mediante el conocimiento y la utilización de estructuras cognitivas. El uso planificado y adaptativo de 
estrategias cognitivas, meta-cognitivas y motivacionales es una habilidad que los profesores podrían ayudar a desarrollar, con estrategias de apoyo que co-regulen este proceso al inicio, mediante el establecimiento de un andamiaje que asegure la aplicación de la autorregulación del aprendizaje (Fuentes Ordaz, Romero Hidalgo y Domínguez Cuenca, 2009). Estos supuestos permiten dar una definición general del aprendizaje autorregulado como un proceso activo constructivo por el cual el aprendiz establece metas para su proceso, y entonces intenta monitorear, regular y controlar su cognición, motivación y conducta, guiados y restringidos a sus metas en las formas contextuales de su ambiente (Pintrich, 2000). Schunk y Zimmerman (2009) hablan de un proceso cíclico por el cual los estudiantes ejercen un control para activar y mantener los pensamientos, conductas y emociones, para alcanzar las metas propuestas.

Un estudio de Zimmerman y Martínez-Pons (1990) sobre el uso de las estrategias autorregulatorias en estudiantes de $5^{\circ}, 8^{\circ}$ y $11^{\circ}$ grado de dos escuelas, una de sobresalientes y otra regular, encontró diferencias en el uso de los planes por sexo, donde las mujeres usan más las estrategias autorregulatorias que los hombres. Por otro lado, estudios de Tejedor-Tejedor, González-González y García-Señoran (2008) concuerdan con los de González-González, García-Señorán, Vargas Jiménez y Cardelle García (2010) sobre las estrategias de recuperación de información y rendimiento en estudiantes de secundaria; confirman que un mayor y mejor uso de éstas incide positivamente en el rendimiento académico en la mayoría de las asignaturas. También observaron que los alumnos del primer ciclo de secundaria utilizan estas estrategias con mayor frecuencia que los de segundo ciclo y, al mismo tiempo, muestran un decremento en el uso de las mismas a medida que aumentan de edad; en el primer estudio se encontró que las mujeres utilizan más las estrategias que los varones.

Algunos autores han propuesto que la actividad de reflexión es tal vez la pieza maestra para establecer el enlace entre meta-cognición y autorregulación y sus posibles interacciones. Reflexión es aquella actividad dinámica que se realiza para sacar inferencias o conclusiones sobre las propias acciones de aprendizaje, y puede efectuarse durante o después de que éstas hayan terminado. A partir de la actividad reflexiva,se puede incrementar el conocimiento meta-cognitivo, refinar las distintas y complejas actividades autorreguladoras y profundizar sobre el propio conocimiento estratégico, para enfrentar con mayor eficacia situaciones posteriores de aprendizaje (Díaz-Barriga y Hernández Rojas, 2002).

El enfoque que sustenta este trabajo es el cognoscitivo, el cual enfatiza que la autorregulación tiene tres fases cíclicas: preparación, que incluye el establecimiento de metas, la elaboración de planes, la autoeficacia y la motivación; desempeño, que implica el autocontrol y la autosupervisión, y proceso de autorreflexión, que incluye la autoevaluación (Woolfolk, 2006).

Este estudio forma parte de la investigación aplicada en una secundaria pública, la cual tiene el objetivo de promover la autorregulación y las estrategias de aprendizaje en el desempeño académico en estudiantes de primer grado de secundaria, mediante un programa de intervención educativa, así como identificar las características de la práctica docente que favorecen estas habilidades. Su principal propósito fue evaluar la influencia de las variables de sexo y edad de los estudiantes, así como la percepción que tienen, tanto del tiempo en el que reciben apoyo en sus tareas escolares, como de los familiares que los apoyan en las mismas con respecto a las estrategias autorregulatorias.

\section{MÉTODO}

\section{Participantes}

Participaron 226 estudiantes del primer grado (el total) de una escuela secundaria pública de Mérida, Yucatán. Adolescentes entre 11 y 14 años de edad, con una media de 12.2 años, provenientes de un nivel socioeconómico bajo y medio bajo, de los cuales 52\% hombres y $48 \%$ mujeres, de los cuales $97.6 \%$ provienen de escuelas primarias públicas.

Instrumento

Se usó una modificación del Cuestionario de Autorregulación de Flores Macías y Cerino Soberanis (2000). Se realizaron análisis de discriminación de reactivos, análisis factoriales y estimaciones de confiabilidad para cada uno de los factores que evalúa el cuestionario, quedando conformado por 48 ítems con cuatro indicadores con cinco opciones de respuesta tipo Likert, que van de 4, Muy parecido a mí, a 0, Nada 
parecido a mí. El cuestionario quedó conformado por cuatro factores:

Factor I. Estrategias de apoyo. Se refiere a aquellos recursos cognitivo-afectivos de los que se vale el estudiante para hacer la tarea. Incluye los enunciados 2, 3, 6, 9, 10, 17, 19, 21, 22, 23, 26, 32, 33, 37, 45, 47 y 54.

Factor II. Estrategias cognitivas. Se refiere al establecimiento de metas y a la aplicación de estrategias cognoscitivas para lograr su aprendizaje. Incluye los enunciados 7, 27, 42 y 49.

Factor III. Estrategias meta-cognitivas. Se refiere al conocimiento de los procesos cognitivos que pone en marcha el estudiante durante el aprendizaje, y el control de los mismos. Incluye los enunciados 5, 12, $13,16,29,34$ y 51.

Factor IV. Motivación. Se refiere a elementos cognitivo-afectivos presentes en todo acto de aprendizaje a los cuales el estudiante atribuye el poder hacer la tarea. Incluye los enunciados $25,28,30,35,40,43,44,52$, y 53, clasificados como positivos, y el 1, 4, 20, 24 y 46, como negativos.

A través del alfa de Cronbach se obtuvieron para cada factor las siguientes confiabilidades: para Estrategias de apoyo, alfa .93; para Estrategias metacognitivas, alfa .84; para Estrategias cognitivas, de .62; y para Motivación de .57 para el elemento positivo y de .79 para el elemento negativo. El alfa de todo el instrumento fue de .88 .

\section{Procedimiento}

Este proyecto es una investigación de carácter aplicado, porque se intervino en una problemática presente en una institución educativa y se realizó en un escenario real; se considera de campo cuasi-experimental porque se aplicó a grupos intactos, utilizando un diseño Pretest-Postest de series de tiempo (Mertens, 2005).

Se administró el cuestionario de autorregulación (medición 1) a todos los estudiantes del primer grado de secundaria, de manera grupal y al inicio del curso escolar; todos los días se realizaron observaciones rigurosas y sistemáticas en los salones de clases para conocer las características de los estudiantes y profesores de cada grupo; se realizó un diagnóstico para identificar las estrategias autorregulatorias en los estudiantes; además, se diseñó y aplicó un programa de intervención en estrategias autorregulatorias a los primeros tres grupos en el primer semestre del curso, para cubrir las necesidades detectadas en sesiones de una hora a la semana durante nueve semanas, cumpliendo así los objetivos del programa. Al término de la intervención con los primeros tres grupos, se hizo la medición 2 para todos los estudiantes de primer año; posteriormente, en el segundo semestre del ciclo escolar, participaron los tres grupos que no recibieron el programa de intervención en autorregulación en las mismas condiciones que los grupos anteriores. Al término del segundo semestre, se hizo la medición 3 para todos los estudiantes del primer grado.

\section{RESULTADOS}

\section{Análisis inferencial}

Con el fin de establecer las diferencias por sexo, se realizó un Análisis de Varianza con prueba post hoc de Scheffé, y un nivel de significancia al .05. Los resultados de este análisis no mostraron diferencias significativas entre mujeres y hombres en el Pretest; sin embargo, en el Postest se encontró una diferencia significativa en el factor Motivación, donde las mujeres presentan un menor nivel (ver Tabla 1).

Tabla 1. Diferencias entre mujeres y hombres en las características autorregulatorias

\begin{tabular}{|c|c|c|c|c|c|c|c|c|}
\hline \multirow{2}{*}{ Factores de Autorregulación } & \multicolumn{2}{|c|}{$M$ Pretest } & \multirow{2}{*}{$F$} & \multirow{2}{*}{$p$} & \multicolumn{2}{|c|}{$M$ Postest } & \multirow{2}{*}{$F$} & \multirow{2}{*}{$p$} \\
\hline & $M$ & $\mathrm{H}$ & & & $M$ & $\mathrm{H}$ & & \\
\hline Motivación & 2.94 & 2.96 & .356 & .551 & 2.86 & 3.02 & 13.514 & .000 \\
\hline Estrategias de apoyo & 3.60 & 3.58 & .020 & .888 & 3.86 & 3.78 & 1.031 & .311 \\
\hline Estrategias meta-cognitivas & 3.48 & 3.23 & 3.425 & .066 & 3.62 & 3.45 & 2.396 & .123 \\
\hline Estrategias cognitivas & 3.06 & 3.10 & .073 & .787 & 3.26 & 3.42 & 1.860 & .174 \\
\hline
\end{tabular}


Asimismo, con el fin de establecer las diferencias por edad (11, 12, 13 y 14 años), se llevó a cabo un Análisis de Varianza con prueba post hoc de Scheffé. Los resultados no mostraron diferencias significativas para esta variable en el Pretest, pero se encontró una diferencia significativa en el Postest para el factor Estrategias de apoyo: $F(3,184)=2.659, p \leq .05$, con los siguientes puntajes: 11 años $M=3.62,12$ años $M=3.88,13$ años $M=3.65$ y 14 años $M=3.49$. Sin embargo, la prueba post hoc de Scheffé no confirmó la significancia.

De acuerdo con la definición de la variable familiar, se tomaron en cuenta los dos valores que la conforman: personas que apoyan y tiempo de apoyo percibido. En ambos casos se llevó a cabo un Análisis de Varianza con prueba post hoc de Scheffé. Para el valor "personas que apoyan" no se encontró diferencia significativa en el Pretest, pero para el Postest, el factor Estrategias cognitivas presentó una diferencia significativa: $F(4,190)=2.609, p \leq .037$, con los siguientes puntajes: Mamá $M=3.48$, Papá $M=3.57$, Ambos $M=$ 3.54 , Otro $M=3.13$ y Nadie $M=3.14$. Aunque la prueba post hoc de Scheffé no confirmó la significancia, el papá juega un papel importante en la adquisición de esta habilidad.

Para el valor "tiempo de apoyo percibido" se encontraron diferencias significativas en el Pretest en el factor Estrategias de apoyo: $F(3,180)=5.398, p \leq .01$, con los siguientes puntajes: Ninguna $M=3.70,1$ hora $\mathrm{M}=3.67,2$ horas $M=4.26$, y Más de 2 horas $\mathrm{M}=3.28$; y en el factor Estrategias cognitivas: $F(3,202)=3.821$, $p \leq .01$, con los siguientes puntajes: Ninguna $M=2.98$, 1 hora $M=3.22$, 2 horas $M=3.71$, y Más de 2 horas $M=$ 2.92. En ambos casos, el tiempo de apoyo percibido " 2 horas" presenta la calificación promedio más elevada. En el Postest, sólo en el factor Estrategias cognitivas se encontró diferencia significativa: $F(3,190)=3.523$, $p$ $\leq .01$, con los siguientes puntajes: Ninguna $M=3.03,1$ hora $M=3.40,2$ horas $M=3.64$, y Más de 2 horas $M=$ 3.43 , resultando ventajoso de nuevo el apoyo percibido de "2 horas" para este factor (ver Figura 1).

\section{DISCUSIÓN}

De acuerdo con los análisis realizados, se puede apreciar que no hubo diferencia entre los hombres y las mujeres en los factores Estrategias de apoyo, Estrategias meta-cognitivas y Estrategias cognitivas; sin embargo, en el factor Estrategias motivacionales las diferencias sí resultaron significativas, ya que las mujeres presentaron un menor grado de motivación comparadas con los hombres. Lo anterior quizá se deba a que en esta población de estudio, por el nivel socioeconómico que prevalece, los padres apoyan más a los hombres para que continúen sus estudios, porque persiste la creencia de que serán los futuros proveedores de sus familias; por el contrario, a las mujeres las apoyan sólo si se esfuerzan lo suficiente en sus logros académicos, porque la creencia es que permanecerán al cuidado de los hijos y serán sólo colaboradoras de la economía familiar. Otro aspecto a considerar es que existen diferencias individuales en la forma de participar de los alumnos en la mayoría de las actividades y tareas escolares, lo que repercute de diferentes maneras en su motivación, como dicen Flores Macías y Gómez Bastida (2010). También hay que tomar en cuenta que la motivación para el aprendizaje requiere un contexto de relaciones favorables, tales como el factor socioeconómico y

Figura 1. Puntajes comparativos significativos del tiempo de apoyo percibido
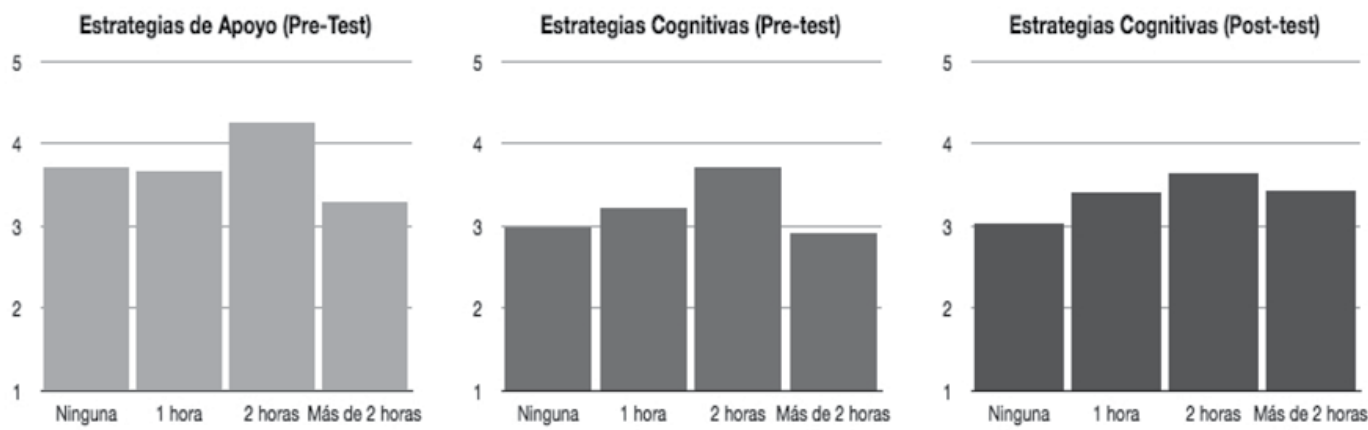
cultural y, por parte de la escuela, la influencia de los profesores, los programas, los sistemas de evaluación, etcétera (Suárez Díaz, 2002).

Asimismo, se puede apreciar que al inicio del curso escolar no hay diferencias en los aspectos autorregulatorios en cuanto a las edades de los estudiantes. Sin embargo, en el Postest en el factor Estrategias de apoyo, los estudiantes de 12 años, edad promedio en este grupo de primer grado, resultaron con ventaja en comparación con las demás edades; de alguna manera los resultados indican que los alumnos del primer ciclo de secundaria utilizan con mayor frecuencia estas estrategias y, al mismo tiempo, muestran un decremento en el uso de las mismas a medida que aumentan de edad, como lo mencionan los estudios de Tejedor-Tejedor et al., (2008) y los de González González et al., (2010).

En el caso de la variable Apoyo familiar, para el valor "personas que apoyan" no se encontró diferencia significativa en el Pretest; pero para el Postest, en el factor Estrategias cognitivas, se presentó una diferencia significativa en el indicador "papá, variable que juega un papel importante en la adquisición de esta habilidad. Estos resultados difieren con los encontrados por Flores Galaz et al., (2008) y Valdés Cuervo et al., (2009), donde a nivel primaria son las madres quienes apoyan más a sus hijos en las actividades escolares en Yucatán; asimismo, en el estudio de Osés Bargas et al., (2009), encontraron que en tercero de secundaria las madres dan más apoyos a sus hijos y son las más interesadas en que continúen con sus estudios. Por otro lado, en la actualidad, tanto la madre como el padre, pasan más tiempo fuera de la casa por motivos laborales. Sin embargo, las madres todavía tienen que realizar las labores domésticas; los padres cuentan con mayor disponibilidad de tiempo, por lo cual podría percibírseles más como elementos de ayuda en estas estrategias. De manera diferencial, se tiene la creencia de que los niños en la primaria, por ser más pequeños, requieren mayor atención de la madre, mientras que en secundaria -por la etapa de desarrollo- lo hijos tienden a distanciarse y a tratar de resolver solos sus problemas. Y, como ya se mencionó, en este nivel socioeconómico los padres apoyan más a los hombres a continuar sus estudios y, tal vez por esa razón, se preocupen más por sus avances académicos.
En relación al valor "tiempo de apoyo percibido", se encontró que en el Pretest en los factores de Estrategias de apoyo y Estrategias cognitivas, cuando se les apoya por dos horas hay diferencias significativas; en el Postest, sólo en el factor Estrategias cognitivas se halló diferencia significativa. Esto se explica porque durante el programa de intervención se hizo énfasis en la organización y la planeación de los tiempos para optimizar el uso de las estrategias, y tener tiempo suficiente para poder practicarlas en casa; y porque en reunión con los padres de familia se exhortó a contar con la ayuda de cualquiera de ellos, o de algún familiar, para ponerlas en práctica; así como también se contó con la colaboración de algunos maestros para insertarlas en su programa educativo. Es en este sentido que se comprueba cómo, con el entrenamiento de las estrategias metacognitivas, se adquiere de forma progresiva el control del aprendizaje que dará lugar a la autonomía (Beltrán Llera, 2004).

En conclusión, se percibe que en relación al sexo no hay diferencias en la forma de adquirir las habilidades autorregulatorias por parte de los estudiantes. No obstante, las mujeres mostraron un decremento en el factor motivacional, que se sugiere investigar si es por la influencia de su cultura de origen o por algún otro factor que podría estar afectándolas. En relación a la edad, se puede apreciar una mejoría en las estrategias de apoyo después de la intervención en los participantes de 12 años; posteriormente se presenta un decremento en el uso de las mismas conforme aumentan los años, lo cual podría explicarse por la crisis de esta etapa de desarrollo donde se preocupan más por los amigos que por su desempeño escolar, como mencionan Papalia et al., (2010).

También es importante enfatizar que la duración de 2 horas del tiempo de apoyo en las tareas escolares juega un papel importante en la adquisición de las habilidades autorregulatorias, y que son los padres los más interesados en el logro de las mismas. Por lo tanto, se comprueba, una vez más, que el uso planificado y adaptativo de estrategias cognitivas, meta-cognitivas y motivacionales es una habilidad que los profesores podrían contribuir a desarrollar mediante el establecimiento de un andamiaje que permita asegurar la aplicación de la autorregulación del aprendizaje (Fuentes Ordaz, Romero Hidalgo y Domínguez Cuenca, 2009). 


\section{REFERENCIAS}

Beltrán Llera, J. (2004). Estrategias de aprendizaje. En E. González y J. A. Bueno (Comps.). Psicología de la educación y del desarrollo en la edad escolar (pp. 679 . 708). España: CCS.

Boekaerts, M., Pintrich, P. \& Zeidner, M. (2000). Handbook of self-regulation. USA: Academic Press.

Castañeda Figueiras, S. \& Ortega, I. (2004). Evaluación de Estrategias de Aprendizaje y Orientación Motivacional, en S. Castañeda Figueiras. (Ed.), Educación, Aprendizaje y Cognición. Teoría en la Práctica. México: Manual Moderno.

Díaz-Barriga, A. F. \& Hernández Rojas, G. (2002). Estrategias docentes para un aprendizaje significativo. Una interpretación constructivista. (2a ed.). México: McGraw Hill.

Flores Galaz, M. M., Cortés Ayala, M. L. \& Góngora Coronado, E. A. (2008). Familia, crianza y personalidad: una perspectiva etnopsicológica. México: Universidad Autónoma de Yucatán.

Flores Macías, R \& Cerino Soberanes, A. (2000). Cuestionario de Autorregulación Académica. México: Universidad Nacional Autónoma de México.

Flores Macías, R. \& Gómez Bastida, J. (2010). Un estudio sobre la motivación hacia la escuela secundaria en estudiantes mexicanos. Revista Electrónica de Investigación Educativa, 12(1). Recuperado el 21 de Marzo de 2011 de http://redie.uabc.mx/vol12no1/ contenido-floresgomez.html.

Fuentes Ordaz, B.; Romero Hidalgo, R. \& Domínguez Cuenca, A. (31 de enero de 2009). Análisis de estrategias de enseñanza que apoyan el aprendizaje autorregulado y significativo de las Matemáticas. Trabajo presentado en el Congreso Internacional para la Investigación y el desarrollo Educativo. Recuperado el 2 de septiembre de 2009 de http://www.colposgrado.edu.mx/congresointernacional/ memorias/fuentes.pdf

González, E. (2006). Desarrollo en la adolescencia. Desarrollo Social y Moral. Problemática en el desarrollo de esta etapa. En E. González y J. A. Bueno. (Comps.), Psicología del Ciclo Vital (pp. 353-398). España: CCS.

González González, S. G., García-Señorán, M. M., Vargas Jiménez, E. \& Cardelle García, F. (2010). Estrategias de recuperación de información y rendimiento en estudiantes de secundaria. Revista de Educación y Desarrollo. 12(enero-marzo). Recuperado el 21 de marzo de 2011 de www.cucs.udg.mx/revistas/edu_ desarrollo/.../012_Gonzalez.pdf

Mertens, D.M. (2005). Research and Evaluation in Education and Psychology. Integrating Diversity with Quantitative, Qualitative, and Mixed Methods (2 ed.). United States of America: Sage Publications, Inc.

Oliva Delgado, A. \& Parra Jiménez, A. (2004). Contexto familiar y desarrollo psicológico durante la adolescencia. En E. Arranz Freijo. (Coordinador). Familia y desarrollo psicológico (pp. 96-122). España: Pearson.

Osés Bargas, R. M., Aguayo Chan, J. C., Duarte Briceño, E. \& Ortega, J. I. (2009). Estrategias de aprendizaje $y$ calidad del desempeño académico en estudiantes de $3^{\circ}$ de secundaria de una escuela pública. Reporte de investigación, no publicado. Universidad Autónoma de Yucatán, México.

Parke, R., Dennis, J., Flyr, M., Morris, K., Leidy, M. \& Schofield, T. (2005). Fathers: Cultural, and Ecological Perspective. En Luster, T. y Okagaki, L. (Eds.), Parenting. An Ecological Perspective. (2ºd., pp 103 144). USA: Lawrence Erlbaum Associates.

Papalia, D. E., Wendkos Olds, S. \& Duskin Feldman, R. (2010). Desarrollo humano. (11º ed.). México: McGraw-Hill.

Pintrich, P. (2000). The Role of Goal Orientation in SelfRegulated Learning. En M. Boekaerts, P. Pintrich, y M. Zeidner. (Eds.), Handbook of Self-Regulation (pp. 451-502). USA: Academic Press.

Schunk, D. H. \& Zimmerman, B. J. (2009). Self regulation of learning and performance. Issues and Educational Applications. USA: Routledge.

Suárez Díaz, R. (2002). La Educación. Estrategias de enseñanza-aprendizaje. Teorías educativas. ( $2^{\mathrm{a}}$ ed.) México: Trillas.

Sternberg, R. \& Baron, J. (1987). Teaching thinking skill. Nueva York: Freeman

Tejedor-Tejedor, F. J.; González-González, S. G. \& GarcíaSeñoran, M. M. (2008). Estrategias Atencionales y Rendimiento Académico en Estudiantes de Secundaria. Revista Latinoamericana de Psicología, 40(1), 123-132. 
Torres Velázquez, L. E. \& Rodríguez Soriano, N. Y. (2006). Rendimiento Académico y contexto familiar en estudiantes universitarios. Revista Enseñanza e Investigación en Psicología, 11(2), julio-diciembre, 255-269.

Valdés Cuervo, A. A., Martín Pavón, M. J. \& Sánchez Escobedo, P.A. (2009). Participación de los padres de alumnos de educación primaria en las actividades académicas de sus hijos. Revista Electrónica de Investigación Educativa, 11(1). Recuperado el 14 de marzo del $2011 \mathrm{de} \mathrm{http://redie.uabc.mx/vol11no1} \mathrm{/conteni-}$ do-valdes.html

Woolfolk, A. (2006). Psicología Educativa. (9o ed.). México: Pearson.

Zimmerman, B. J. \& Martínez-Pons, M. (1990). Student Differences in Self-Regulated Learning: Relating Grade, Sex, and Giftedness to Self-Efficacy and Strategy Use. Journal of Educational Psychology, 82(1), 51-59. 